\section{Using bar codes to create a robust national vaccine registry}

I thank Dr. Hapuhennedige for the informative article published in CMAJ, "Vaccination debates may obscure access issues."

Canada continues to lack a tracking system for adult vaccines. Instead, provinces and territorial public health records contain vaccination records for children only. ${ }^{2}$ Data on vaccines provided to adults in all settings are missing, including on vaccines administered in clinicians' offices, private pharmacies, long-term care homes, prisons, First Nation reserves and given to adult newcomers to Canada.

Yet it is adults, especially those older than 60 years and with chronic conditions, who are most at risk of hospital admission for and death from COVID-19.

Now that vaccines for severe acute respiratory syndrome coronavirus 2 (SARSCoV-2) are being developed, the lack of an adult vaccine registry has been further highlighted and may be compounded by vaccine shortages. It is imperative that we have the ability to optimize vaccine distribution and coverage to places and people where need is greatest.

Using bar codes to create such registries would prove indispensable in this effort. Standardized bar codes are currently found on nearly all commonly administered vaccines and contain the vaccine's name, lot number and expiry date.

Patients would need to be electronically assigned a unique bar code if one doesn't already exist. Ontario already has such a bar code on its health cards.

In this model, the patient's bar code would be scanned, along with that of the vaccine being provided at all locations, including pharmacies, offices and health units.
Once scanned, 3 records would be simultaneously updated, including the following:

- Patients' electronic health record, which saves clinician entry time and time needed to reorder vaccines, reduces manual transcription errors ${ }^{3}$ and reduces the provision of unnecessary vaccines ${ }^{4}$

- Local Public Health Unit's record, which automates vaccine supply to offices and reduces vaccine wastage by better matching quantities delivered to those actually used

- Provincial and territorial vaccine registry with features that include the following: a patient portal to increase patient autonomy, automated email reminders to improve adherence to vaccine schedules ${ }^{5}$ and educational resources on vaccines, including addressing vaccine hesitancy.

The universal utilization of scanning bar codes would benefit Ministries of Health by allowing a comparison of vaccine coverage with disease rates within specified areas, optimizing the distribution of scarce vaccines during a pandemic or when faced with vaccine shortages, more easily recalling vaccine lots that have been contaminated, and redistributing vaccines nearing expiry to locations with immediate need.

Using bar codes has been shown to save time, improve user experience, simplify documentation and reduce transcription errors, and would create robust and comprehensive provincial and territorial vaccine registries.

Critically robust registries of this kind, once anonymized, would form the building blocks for a National Immunization Registry, a long-sought-after goal of public health.

\section{Iris Gorfinkel MD CM}

General practitioner, vaccine researcher, founder and principal investigator, PrimeHealth Clinical Research, Toronto, Ont.

- Cite as: CMAJ 2021 March 8;193:E344. doi: $10.1503 / \mathrm{cmaj} .76626$

\section{References}

1. Hapuhennedige S. Vaccination debates may obscure access issues. CMAJ 2020;192:E935-6.

2. Vaccination coverage in Canada. Ottawa: Government of Canada; 2019. Available: www.canada.ca /en/public-health/services/immunization-vaccines /vaccination-coverage.html (accessed 2020 Aug. 10).

3. PHAC, GS1 Canada, and vaccine industry approve major vaccine identification initiative: Longwoods.com [Health \& Healthcare News]; 2010 Nov. 10. Available: www.longwoods.com/ newsdetail/1590/print (accessed 2020 Aug. 10).

4. Evanson HV, Rodgers L, Reed J, et al. Experience and compliance with scanning vaccines' twodimensional barcodes to record data. Comput Inform Nurs 2018;36:8-17.

5. Crowcroft NS, Levy-Bruhl D. Registries: an essential tool for maximising the health benefits of immunisation in the 21st century. Euro Surveill 2017;22:30523.

Competing interests: Iris Gorfinkel has participated in more than 60 clinical research trials, funded by several major pharmaceutical companies and the National Institute of Health. Dr. Gorfinkel has received funding from GlaxoSmithKline and Bayer Pharmaceuticals for educational programming. She served as the co-chair of the advisory board for Shingrix, manufactured by GlaxoSmithKline.

Content licence: This is an Open Access article distributed in accordance with the terms of the Creative Commons Attribution (CC BY-NC-ND 4.0) licence, which permits use, distribution and reproduction in any medium, provided that the original publication is properly cited, the use is noncommercial (i.e., research or educational use), and no modifications or adaptations are made. See: https://creativecommons.org/ licenses/by-nc-nd/4.0/ 